\title{
Asymmetric Mannich-type Reactions of Fluorinated Ketoesters with Binaphthyl-Modified Thiourea Catalysts
}

\author{
Young Ku Kang, Sung Je Yoon, and Dae Young Kim* \\ Department of Chemistry, Soonchunhyang University, Asan, Chungnam 336-745, Korea. *E-mail: dyoung@sch.ac.kr \\ Received December 31, 2010, Accepted February 4, 2011
}

\begin{abstract}
The catalytic enantioselective Mannich-type reaction promoted by chiral binaphthyl-modified bifunctional organocatalysts is described. The treatment of $\alpha$-fluoro- $\beta$-ketoesters with $N$-Boc imines under mild reaction conditions afforded the corresponding $\beta$-aminated $\alpha$-fluoro- $\beta$-ketoesters with excellent enantioselectivities (up to $98 \%$ ee).
\end{abstract}

Key Words : Mannich reaction, Asymmetric catalysis, Bifunctional organocatalyst, $\alpha$-Fluoro- $\beta$-ketoesters, $N$ Boc imines

\section{Introduction}

Fluorinated molecules are of high importance in medicinal and organic chemistry. ${ }^{1}$ Introduction of fluorine atom into biologically active compounds often leads to improvement of their biological characteristics due to unique properties of the fluorine atom. ${ }^{2}$ Enantiopure fluorine-containing organic molecules are interesting and important materials with uses in analytical, biological, and medicinal chemistry and also in the chemistry of polymers and materials. ${ }^{3}$ Among various strategies, electrophilic fluorination of active methines and $\mathrm{C}-\mathrm{C}$ bond formation of fluorocarbon nucleophiles are two typical approaches for the construction of fluorine-containing molecules, and their asymmetric versions are particularly attractive. Enantioselective electrophilic fluorination has been achieved with the aid of electrophilic fluorinating agents using chiral transition-metal catalysts and organocatalysts with excellent enantioselectivities. ${ }^{4,5}$ On the other hand, the use of fluorinated active methine nucleophiles such as fluoromalonate, ${ }^{6} \alpha$-fluoro- $\beta$-ketoesters, ${ }^{7}$ and fluorobis(phenylsulfonyl)methane ${ }^{8}$ for a catalytic asymmetric reaction has become increasingly popular. Several groups have developed organocatalytic conjugate addition of nucleophilic $\alpha$-fluoro- $\beta$-ketoesters to nitroalkenes, ${ }^{7 \mathrm{~d}-\mathrm{f}}$ Michael reaction to $\alpha, \beta$-unsaturated ketone, ${ }^{7 \mathrm{~h}}$ phase-transfer catalytic amination to azodicarboxylate. ${ }^{7 b}$ Enantioselective Mannich reactions are efficient and powerful methods to prepare chiral $\beta$-amino carbonyl derivatives. ${ }^{9}$ Tremendous efforts have been made in the development of efficient chiral catalysts for enantioselective Mannich-type reactions with preformed enolates $^{10}$ and enolizable $\beta$-dicarbonyl and related compounds. ${ }^{11}$ Recently, Lu and Tan groups report the catalytic enantioselective Mannich reactions of $\alpha$-fluoro- $\beta$-ketoesters with $N$-Boc imines using tryptophan-derived organocatalyst and chiral bicyclic guanidine catalyst. ${ }^{12}$

As part of a research program related to the development of synthetic methods for the enantioselective construction of stereogenic carbon centers, ${ }^{13}$ we recently reported chiral amine-thiourea bifunctional organocatalyst to be a highly selective catalyst for the enantioselective addition reaction of active methines. ${ }^{14}$ We envisioned that the assembly of a structurally well-defined chiral 1,2-diamine and binaphthyl scaffold with a thiourea motif could constitute a new class of bifunctional organocatalyst. The rigid binaphthyl structure can serve as an efficient stereocontrolling axial chiral element. In this article, we wish to describe the direct enantioselective Mannich reaction of $\alpha$-fluoro- $\beta$-ketoesters with simple $N$ Boc imines catalyzed by binaphthyl-modified thiourea organocatalysts bearing both central and axial chiral elements.

\section{Results and Discussion}

The ogranocatalysts I-VIII (Fig. 1) were prepared according to the reported procedure. ${ }^{14,15}$ In an attempt to validate the feasibility of the organocatalytic enantioselective Mannich reaction of $\alpha$-fluoro- $\beta$-ketoesters, we initially investigated the reaction system with $\alpha$-fluoro- $\beta$-ketoester $1 \mathbf{a}$ and $N$-Boc $p$-tolualdimine (2a) in the presence of $10 \mathrm{~mol} \%$ of catalyst in toluene at room temperature. We first examined the impact of the structure of catalysts I-VIII on enantioselectivities (Table 1, entries 1-8).

While chiral bifunctional organocatalysts I-VIII bearing both central and axial chiral elements or cinchona alkaloid backbone effectively promoted the addition in high yield but with moderate enantioselectivity $(8-76 \%$ ee), Catalyst III gave the desired product 3a with high enantioselectivity (entry 3). Based on the exploratory studies, we decided to select catalyst III for further optimization of reaction conditions. A survey of the reaction media indicated that many common solvents, such as toluene, dichloromethane, THF, diethyl ether, tert-butyl methyl ether, diphenyl ether, and ethyl vinyl ether (entries 3 and 9-14), were well tolerated in this Mannich-type reaction with moderate to high enantioselectivities. Among the solvents probed, the best results (97\% yield, 85:15 dr, and 85\% ee) were achieved when the reaction was conducted in diethyl ether (entry 14). Lowering the temperature to $-40{ }^{\circ} \mathrm{C}$ with catalyst III improved the diastereoselectivty, but decreased slightly the enantioselec- 
<smiles>C=CC1CC2CCN1C2C(NC(=S)Nc1cc(C(F)(F)F)cc(C(F)(F)F)c1)c1ccnc2ccc(OC)cc12</smiles><smiles>O=C(NC1CCCCC1NC(=S)Nc1cc(C(F)(F)F)cc(C(F)(F)F)c1)NC1CCCCC1N1Cc2ccc3ccccc3c2-c2c(ccc3ccccc23)C1</smiles>

V<smiles>FC(F)(F)c1cc(NC(=S)NC(c2ccccc2)C(c2ccccc2)N2Cc3ccc4ccccc4c3-c3c(ccc4ccccc34)C2)cc(C(F)(F)F)c1</smiles>

II<smiles>O=S(=O)(NC1CCCCC1NC(=S)NC1CCCCC1N1Cc2ccc3ccccc3c2-c2c(ccc3ccccc23)C1)c1cc(C(F)(F)F)cc(C(F)(F)F)c1</smiles>

VI<smiles>C/C=C\C=C/CCC</smiles>

III<smiles>O=c1c(NCc2cc(C(F)(F)F)cc(C(F)(F)F)c2)c(NC2CCCCC2[NH+]2Cc3ccc4ccccc4c3-c3c(ccc4ccccc34)C2)c1=O</smiles>

VII<smiles>O=C(Nc1cc(C(F)(F)F)cc(C(F)(F)F)c1)N[C@H]1CCCC[C@H]1N1Cc2ccc3ccccc3c2-c2c(ccc3ccccc23)C1</smiles>

IV

Figure 1. Structures of various chiral thiourea-tertiary amine catalysts.

Table 1. Optimization of the reaction conditions

\begin{tabular}{|c|c|c|c|c|c|}
\hline Entry & Cat. & Solvent & Yield $(\%)^{a}$ & $\mathrm{dr}(\%)^{b}$ & ee $(\%)^{c}$ \\
\hline 1 & I & $\mathrm{PhMe}$ & 97 & $80: 20$ & 45 \\
\hline 2 & II & PhMe & 32 & $67: 36$ & 8 \\
\hline 3 & III & $\mathrm{PhMe}$ & 97 & $77: 23$ & 76 \\
\hline 4 & IV & PhMe & 98 & $88: 22$ & 37 \\
\hline 5 & V & PhMe & 60 & $69: 31$ & 45 \\
\hline 6 & VI & $\mathrm{PhMe}$ & 48 & $87: 13$ & 16 \\
\hline 7 & VII & $\mathrm{PhMe}$ & 60 & $50: 50$ & 24 \\
\hline 8 & VIII & $\mathrm{PhMe}$ & 69 & $77: 23$ & 63 \\
\hline 9 & III & DCM & 64 & $82: 18$ & 63 \\
\hline 10 & III & THF & 27 & $79: 21$ & 30 \\
\hline 11 & III & TBME & 98 & $79: 21$ & 62 \\
\hline 12 & III & $\mathrm{Ph}_{2} \mathrm{O}$ & 98 & $81: 19$ & 68 \\
\hline 13 & III & $\mathrm{EtOCH}=\mathrm{CH}_{2}$ & 98 & $79: 21$ & 85 \\
\hline 14 & III & $\mathrm{Et}_{2} \mathrm{O}$ & 97 & $85: 15$ & 88 \\
\hline $15^{d}$ & III & $\mathrm{Et}_{2} \mathrm{O}$ & 68 & $90: 10$ & 84 \\
\hline $16^{e}$ & III & $\mathrm{Et}_{2} \mathrm{O}$ & 67 & 93:7 & 82 \\
\hline $17^{f}$ & III & $\mathrm{Et}_{2} \mathrm{O}$ & 60 & $96: 4$ & 79 \\
\hline
\end{tabular}

${ }^{a}$ Isolated yield. ${ }^{b}$ The diastereomeric ratio was determined by ${ }^{1} \mathrm{H}$ NMR spectroscopic analysis of the crude product. ${ }^{c}$ Enantiomeric excess of major diastereomer was determined by HPLC analysis using Chiralpak $\mathrm{AD}-\mathrm{H}$ column. ${ }^{d}$ This reaction was carried out at $0{ }^{\circ} \mathrm{C}$. ${ }^{e} \mathrm{This}$ reaction was carried out at $-20^{\circ} \mathrm{C}$. ${ }^{f}$ This reaction was carried out at $-40{ }^{\circ} \mathrm{C}$.
Table 2. Variation of $N$-Boc imines

\begin{tabular}{|c|c|c|c|c|}
\hline & 2 & & & \\
\hline Entry & 2, Ar & Yield $(\%)^{a}$ & $\operatorname{dr}(\%)^{b}$ & ee $(\%)^{c}$ \\
\hline 1 & 2a, $p-\mathrm{MeC}_{6} \mathrm{H}_{4}$ & 3a, 98 & $83: 17$ & 88 \\
\hline 2 & 2b, $p-\mathrm{BrC}_{6} \mathrm{H}_{4}$ & $\mathbf{3 b}, 80$ & $85: 15$ & 89 \\
\hline 3 & $\mathbf{2 c}, p-\mathrm{ClC}_{6} \mathrm{H}_{4}$ & 3c, 98 & $88: 12$ & 81 \\
\hline 4 & 2d, $\mathrm{C}_{6} \mathrm{H}_{5}$ & 3d, 84 & $91: 9$ & 88 \\
\hline 5 & $2 \mathrm{e}, o-\mathrm{ClC}_{6} \mathrm{H}_{4}$ & 3e, 98 & $>99: 1$ & 86 \\
\hline 6 & 2f, $o-\mathrm{FC}_{6} \mathrm{H}_{4}$ & 3f, 88 & 99:1 & 76 \\
\hline 7 & $2 \mathbf{g}, m-\mathrm{NO}_{2} \mathrm{C}_{6} \mathrm{H}_{4}$ & $3 g, 80$ & $83: 7$ & 79 \\
\hline 8 & 2h, 1-naphthyl & 3h, 90 & 91:9 & 81 \\
\hline 9 & 2i, 2-furyl & $3 \mathbf{i}, 98$ & $73: 27$ & 98 \\
\hline 10 & 2j, 2-thienyl & $3 \mathbf{j}, 78$ & $95: 5$ & 95 \\
\hline
\end{tabular}

${ }^{a}$ Isolated yield. ${ }^{b}$ The diastereomeric ratio was determined by ${ }^{1} \mathrm{H}$ NMR spectroscopic analysis of the crude product. ${ }^{c}$ Enantiomeric excess of major diastereomer was determined by HPLC analysis using Chiralpak AD-H (for 3a), IA (for 3b-g, 3i-j), Whelk-O1 (for 3h) columns.

tivity (entries 14-17). Absolute configuration of major diastereomer of 3a was determined to be $(2 S, 3 S)$ by comparison of the optical rotation and chiral HPLC data with the literature values. $^{12}$

We then explored the possibility of using wide range of $\mathrm{N}$ Boc protected substituted aromatic and heteroaromatic imines 2 with $\alpha$-fluoro- $\beta$-ketoester 1a under the optimized reaction condition. As shown in Table 2, high yields (78-98\%), 
Table 3. Variation of $\alpha$-fluoro- $\beta$-ketoesters

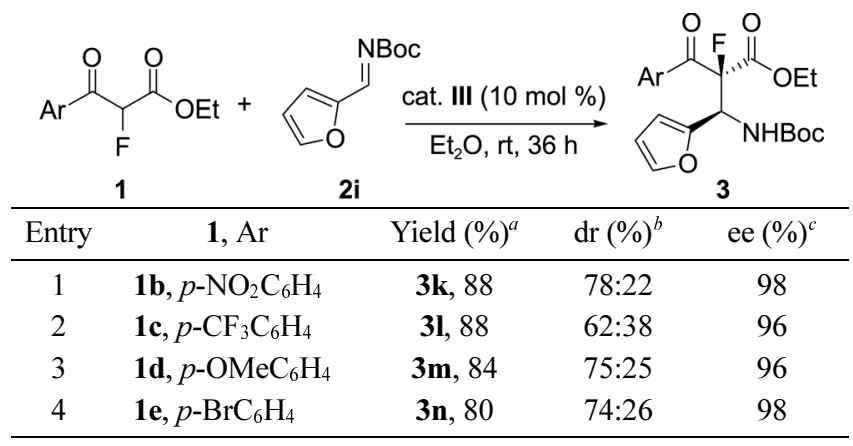

${ }^{a}$ Isolated yield. ${ }^{b}$ The diastereomeric ratio was determined by ${ }^{1} \mathrm{H}$ NMR spectroscopic analysis of the crude product. ${ }^{c}$ Enantiomeric excess of major diastereomer of 3k-n was determined by HPLC analysis using Chiralpak IA column.

excellent diastereoselectivities (up to $>99: 1$ ), and excellent enantioselectivities (up to $98 \%$ ) were observed for different substitution patterns on the aromatic ring of imines 2. Both electron-withdrawing and electron-donating substrates, as well as the heteroaromatic substrates gave excellent results.

To examine the generality of the catalytic enantioselective Mannich-type reaction of $\alpha$-fluoro- $\beta$-ketoesters 1 by using naphthyl-modified bifunctional organocatalyst III, we studied the addition of various $\alpha$-fluoro- $\beta$-ketoesters 1 to $N$-Boc aldimine $2 \mathbf{i}$. As it can be seen by the results summarized in Table 3, the corresponding products 3k-n were obtained in high to excellent yields (80-88\%), high diastereoselectivities (62:38-78:22), and excellent enantioselectivities (96-98\%).

We examined the direct enantioselective Mannich-type reaction of $\alpha$-fluoro acetoacetate derivatives $1 \mathbf{k}-\mathbf{l}$ with $N$ Boc $p$-tolualdimine (2a) using bifunctional organocatalyst III in diethyl ether at room temperature. In the presence of $10 \mathrm{~mol} \%$ of catalyst III, the reaction proceeded to afford the $\beta$-aminated product 3o-p after $36 \mathrm{~h}$ with $72-80 \%$ ee (Scheme 1).

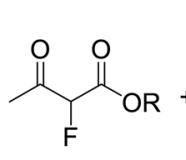

$1 \mathbf{k}, \mathrm{R}=\mathrm{Et}$ 1I, $\mathrm{R}=\mathrm{Bn}$

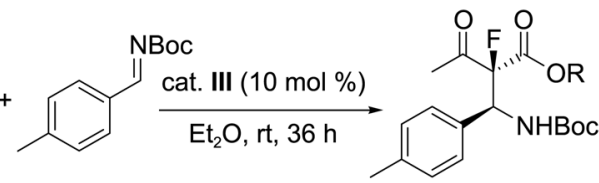

$2 a$

3o, $88 \%, 67: 33 \mathrm{dr}, 80 \%$ ee $3 p, 64 \%, 87: 13 \mathrm{dr}, 72 \%$ ee
Scheme 1

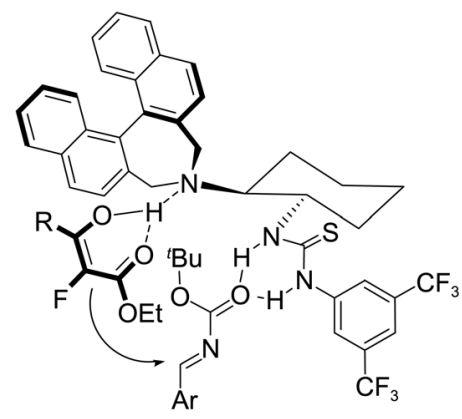

Figure 2. Proposed stereochemical model.
Although the reason for the observed enantioselectivity is still unclear, we believe that a carbonyl group of the $\mathrm{N}$ Boc imine $\mathbf{2}$ is activated by the thiourea moiety through hydrogen bonding, and the $\alpha$-fluoro- $\beta$-ketoester moiety is activated by the basic nitrogen atom in tertiary amine (Fig. 2 ). These interactions control the stereochemical outcome of the reaction and increase the reaction rate.

\section{Conclusion}

In conclusion, we have developed a highly efficient catalytic enantioselective Mannich reaction of $\alpha$-fluoro- $\beta$-ketoesters using chiral binaphthyl-modified thiourea organocatalysts. The desired $\beta$-aminated products were obtained in good to high yields, and high enantioselectivities (72-98\% ee) were observed for all the substrates examined in this work. We believe that this method provides a practical entry for the preparation of chiral $\beta$-aminated $\alpha$-fluoro- $\beta$-ketoester derivatives. Further study of these bifunctional organocatalysts in other asymmetric reactions is being under investigated.

\section{Experimental Section}

General. All commercial reagents and solvents were used without purification. TLC analyses were carried out on precoated silica gel plates with $\mathrm{F}_{254}$ indicator. Visualization was accomplished by UV light (254 nm), I $2, p$-anisaldehyde, ninhydrin, and phosphomolybdic acid solution as an indicator. Purification of reaction products was carried out by flash chromatography using E. Merck silica gel 60 (230-400 mesh). ${ }^{1} \mathrm{H}$ NMR and ${ }^{13} \mathrm{C}$ NMR spectra were recorded on a Bruker AC 200 (200 MHz for ${ }^{1} \mathrm{H}, 50 \mathrm{MHz}$ for $\left.{ }^{13} \mathrm{C}\right)$. Chemical shift values $(\delta)$ are reported in ppm relative to $\mathrm{Me}_{4} \mathrm{Si}(\delta 0.0$ ppm). Optical rotations were measured on a JASCO-DIP1000 digital polarimeter with a sodium lamp. The enantiomeric excesses (ee's) were determined by HPLC. HPLC analysis was performed on Younglin M930 Series and Younglin M720 Series, measured at $254 \mathrm{~nm}$ using the indicated chiral column.

General Procedure for the Mannich-type Reaction of $\alpha$-fluoro- $\beta$-ketoester 1 with $\boldsymbol{N}$-Boc Imine 2: To a solution of $\alpha$-fluoro- $\beta$-ketoester $(\mathbf{1}, 0.3 \mathrm{mmol})$ and catalyst III $(0.03$ $\mathrm{mmol}, 20 \mathrm{mg})$ in $\mathrm{Et}_{2} \mathrm{O}(6 \mathrm{~mL})$ was added $N$-Boc imine $(2$, $0.45 \mathrm{mmol}$ ). Reaction mixture was stirred for $24-36 \mathrm{~h}$ at room temperature, concentrated, and purified by flash column chromatography (EtOAc/hexane:1/7) to afford the Mannich adduct 3 .

(2S, 3S)-Ethyl 2-Benzoyl-3-(tert-butoxycarbonylamino)2-fluoro-3-p-tolylpropanoate (3a). Major diastereomer: $[\alpha]_{\mathrm{D}}^{31}=20.0\left(c=1.0, \mathrm{CHCl}_{3}\right) ;{ }^{1} \mathrm{H} \mathrm{NMR}\left(200 \mathrm{MHz}, \mathrm{CDCl}_{3}\right)$ $\delta 1.28(\mathrm{t}, J=6.9 \mathrm{~Hz}, 3 \mathrm{H}), 1.39(\mathrm{~s}, 9 \mathrm{H}), 2.26(\mathrm{~s}, 3 \mathrm{H}), 4.18-$ $4.39(\mathrm{~m}, 2 \mathrm{H}), 5.44(\mathrm{~d}, J=10.4 \mathrm{~Hz}, 1 \mathrm{H}), 5.96\left(\mathrm{dd},{ }^{2} J=28.9\right.$ $\left.\mathrm{Hz},{ }^{1} \mathrm{~J}=10.4 \mathrm{~Hz}, 1 \mathrm{H}\right), 7.04-7.08$ (m, 2H), 7.26-7.29 (m, 2H), 7.34-7.39 (m, 2H), 7.49-7.54 (m, 1H), 7.81-7.84 (m, 2H); ${ }^{13} \mathrm{C}$ NMR (50 MHz, $\left.\mathrm{CDCl}_{3}\right) \delta 13.77,20.96,28.11,57.11(\mathrm{~d}$, $J=18.35 \mathrm{~Hz}), 63.01,79.96,102.21(\mathrm{~d}, J=203.75 \mathrm{~Hz})$, 
$128.35,128.56,128.96,129.33,129.46,133.54,133.66$, $137.71,154.29,165.49$ (d, $J=26.9 \mathrm{~Hz}), 190.76$ (d, $J=25.6$ $\mathrm{Hz}$ ); HPLC (80:20, $n$-hexane : $i$-PrOH, $254 \mathrm{~nm}, 1.0 \mathrm{~mL} /$ min) Chiralpak AD-H column, $t_{\mathrm{R}}=10.4$ min (minor), $t_{\mathrm{R}}=$ 14.9 min (major), $88 \%$ ee.

(2S, 3S)-Ethyl 2-Benzoyl-3-(tert-butoxycarbonylamino)2-fluoro-3-(4-bormophenyl)propanoate (3b). Major diastereomer: $[\alpha]_{\mathrm{D}}^{31}=37.3\left(c=1.0, \mathrm{CHCl}_{3}\right) ;{ }^{1} \mathrm{H}$ NMR $(200$ $\left.\mathrm{MHz}, \mathrm{CDCl}_{3}\right) \delta 1.27(\mathrm{t}, J=7.0 \mathrm{~Hz}, 3 \mathrm{H}), 1.38(\mathrm{~s}, 9 \mathrm{H}), 4.17-$ $4.41(\mathrm{~m}, 2 \mathrm{H}), 5.48(\mathrm{~d}, J=10.4,1 \mathrm{H}), 5.95\left(\mathrm{dd},{ }^{1} J=28.7 \mathrm{~Hz}\right.$, $\left.{ }^{2} J=10.4 \mathrm{~Hz}, 1 \mathrm{H}\right), 7.27-7.48(\mathrm{~m}, 5 \mathrm{H}), 7.51-7.59(\mathrm{~m}, 2 \mathrm{H})$, 7.82-7.86 (m, 2H); ${ }^{13} \mathrm{C} \mathrm{NMR}\left(50 \mathrm{MHz}, \mathrm{CDCl}_{3}\right) \delta 13.79$, 28.11, 56.80 (d, $J=18.2 \mathrm{~Hz}), 63.29,80.30,101.93$ (d, $J=$ 204.0 Hz), 122.23, 128.50, 129.41, 129.53, 130.54, 131.43, $134.00,135.63,154.24,165.22$ (d, $J=27.2 \mathrm{~Hz}), 190.39$ (d, $J$ $=25.1 \mathrm{~Hz}$ ); HPLC (85: 15, $n$-hexane : EtOH, $254 \mathrm{~nm}, 0.5$ $\mathrm{mL} / \mathrm{min}$ ) Chiralpak IA column, $t_{\mathrm{R}}=15.8 \mathrm{~min}$ (major), $t_{\mathrm{R}}=$ $17.3 \mathrm{~min}$ (minor), $89 \%$ ee.

(2S, 3S)-Ethyl 2-Benzoyl-3-(tert-butoxycarbonylamino)2-fluoro-3-(4-chlorophenyl)propanoate (3c). Major diastereomer: $[\alpha]_{\mathrm{D}}^{26}=7.0\left(c=1.0, \mathrm{CHCl}_{3}\right) ;{ }^{1} \mathrm{H}$ NMR $(200$ $\left.\mathrm{MHz}, \mathrm{CDCl}_{3}\right) \delta 1.28(\mathrm{t}, J=6.9 \mathrm{~Hz}, 3 \mathrm{H}), 1.39(\mathrm{~s}, 9 \mathrm{H}), 4.18-$ $4.41(\mathrm{~m}, 2 \mathrm{H}), 5.45(\mathrm{~d}, J=10.4 \mathrm{~Hz}, 1 \mathrm{H}), 5.87(\mathrm{dd}, J=28.8$, $10.4 \mathrm{~Hz}, 1 \mathrm{H}), 7.35-7.44(\mathrm{~m}, 5 \mathrm{H}), 7.54-7.58$ (m, 2H), 7.80$7.84(\mathrm{~m}, 2 \mathrm{H}) ;{ }^{13} \mathrm{C}$ NMR $\left(50 \mathrm{MHz}, \mathrm{CDCl}_{3}\right) \delta 13.82,28.15$, $56.78(\mathrm{~d}, J=17.7 \mathrm{~Hz}), 63.32,80.35,102.01$ (d, $J=204.4$ $\mathrm{Hz}), 128.54,129.56,129.81,130.24 \times 2,134.01,135.12$, 154.30, 165.32 (d, $J=26.5 \mathrm{~Hz}), 190.33$ (d, $J=25.7 \mathrm{~Hz})$; HPLC (85 : 15, $n$-hexane : $i$-PrOH, $254 \mathrm{~nm}, 0.5 \mathrm{~mL} / \mathrm{min})$ Chiralpak IA column, $t_{\mathrm{R}}=23.8 \mathrm{~min}$ (minor), $t_{\mathrm{R}}=28.1 \mathrm{~min}$ (major), $81 \%$ ee.

(2S, 3S)-Ethyl 2-Benzoyl-3-(tert-butoxycarbonylamino)2-fluoro-3-propanoate (3d). Major diastereomer: $[\alpha]_{\mathrm{D}}^{29}=$ $39.3\left(c=1.0, \mathrm{CHCl}_{3}\right) ;{ }^{1} \mathrm{H} \mathrm{NMR}\left(200 \mathrm{MHz}, \mathrm{CDCl}_{3}\right) \delta 1.26(\mathrm{t}$, $J=6.8 \mathrm{~Hz}, 3 \mathrm{H}), 1.38(\mathrm{~s}, 9 \mathrm{H}), 4.16-4.39(\mathrm{~m}, 2 \mathrm{H}), 5.57$ (d, $J=$ $10.5 \mathrm{~Hz}, 1 \mathrm{H}), 6.02$ (dd, $J=28.8,10.5 \mathrm{~Hz}, 1 \mathrm{H}), 7.19-7.33$ (m, 4H), 7.36-7.51 (m, 4H), 7.80-7.83 (m, 2H); ${ }^{13} \mathrm{C}$ NMR (50 $\left.\mathrm{MHz}, \mathrm{CDCl}_{3}\right) \delta 13.75,28.08,57.38(\mathrm{~d}, J=18.5 \mathrm{~Hz}), 63.11$, 79.97, 102.19 (d, $J=204 \mathrm{~Hz}), 128.01,128.26,128.34$, $128.74,129.28,129.39,133.69,136.50,154.31,165.38$ (d, $J$ $=27.1 \mathrm{~Hz}), 190.80(\mathrm{~d}, J=25.6 \mathrm{~Hz})$; HPLC $(85: 15, n-$ hexane : $i-\mathrm{PrOH}, 254 \mathrm{~nm}, 0.5 \mathrm{~mL} / \mathrm{min}$ ) Chiralpak IA column, $t_{\mathrm{R}}=23.5 \mathrm{~min}$ (minor), $t_{\mathrm{R}}=31.0 \mathrm{~min}$ (major), $88 \%$ ee.

(2S, 3S)-Ethyl 2-Benzoyl-3-(tert-butoxycarbonylamino)2-fluoro-3-(2-chlorophenyl)propanoate (3e). Major diastereomer: $[\alpha]_{\mathrm{D}}^{28}=55.5\left(c=1.0, \mathrm{CHCl}_{3}\right) ;{ }^{1} \mathrm{H}$ NMR $(200$ $\left.\mathrm{MHz}, \mathrm{CDCl}_{3}\right) \delta 1.28(\mathrm{t}, J=6.1 \mathrm{~Hz}, 3 \mathrm{H}), 1.39(\mathrm{~s}, 9 \mathrm{H}), 4.23-$ $4.35(\mathrm{~m}, 2 \mathrm{H}), 5.55(\mathrm{~d}, J=10.1 \mathrm{~Hz}, 1 \mathrm{H}), 6.47(\mathrm{dd}, J=26.1$, $10.1 \mathrm{~Hz}, 1 \mathrm{H}), 7.10-7.18$ (m, 2H), 7.33-7.55 (m, 5H), 7.87$7.91(\mathrm{~m}, 2 \mathrm{H}) ;{ }^{13} \mathrm{C} \mathrm{NMR}\left(50 \mathrm{MHz}, \mathrm{CDCl}_{3}\right) \delta 13.53,27.88$, $53.62(\mathrm{~d}, J=18.7 \mathrm{~Hz}$ ), 63.02, 79.84, 101.30 (d, $J=205.4$ $\mathrm{Hz}), 126.76,128.30,128.85,129.09,129.22,129.85 \times 2$, $133.71,134.57,134.97,153.89,165.15$ (d, $J=26.7 \mathrm{~Hz})$, $190.22(\mathrm{~d}, J=24.9 \mathrm{~Hz})$; HPLC ( $85: 15, n$-hexane : $i$-PrOH, $254 \mathrm{~nm}, 0.5 \mathrm{~mL} / \mathrm{min}$ ) Chiralpak IA column, $t_{\mathrm{R}}=30.4 \mathrm{~min}$ (minor), $t_{\mathrm{R}}=51.2 \mathrm{~min}$ (major), $86 \%$ ee.

(2S, 3S)-Ethyl 2-Benzoyl-3-(tert-butoxycarbonylamino)- 2-fluoro-3-(2-fluorophenyl)propanoate (3f). Major diastereomer: $[\alpha]_{\mathrm{D}}^{30}=43.5\left(c=1.0, \mathrm{CHCl}_{3}\right) ;{ }^{1} \mathrm{H}$ NMR $(200$ $\left.\mathrm{MHz}, \mathrm{CDCl}_{3}\right) \delta 1.27$ (t, $\left.J=7.3 \mathrm{~Hz}, 3 \mathrm{H}\right), 1.39$ (s, 9H), 4.22$4.38(\mathrm{~m}, 2 \mathrm{H}), 5.64(\mathrm{~d}, J=10.4 \mathrm{~Hz}, 1 \mathrm{H}), 6.25\left(\mathrm{dd},{ }^{1} J=28.4\right.$ $\left.\mathrm{Hz},{ }^{2} J=10.4 \mathrm{~Hz}, 1 \mathrm{H}\right), 6.97-7.07(\mathrm{~m}, 2 \mathrm{H}), 7.18-7.26(\mathrm{~m}, 1 \mathrm{H})$, 7.34-7.42 (m, 3H), 7.47-7.57 (m, 1H), 7.85-7.90 (m, 2H); ${ }^{13} \mathrm{C}$ NMR $\left(50 \mathrm{MHz}, \mathrm{CDCl}_{3}\right) \delta 13.80,28.13,52.85(\mathrm{~d}, J=$ 19.6 Hz), 63.24, 80.20, 101.92 (d, $J=204.7 \mathrm{~Hz}), 115.89$ (d, $J=22.7 \mathrm{~Hz}), 123.5(\mathrm{~d}, J=12.1 \mathrm{~Hz}), 124.02(\mathrm{~d}, J=6.45 \mathrm{~Hz})$, 128.48, 129.44, 129.56, $129.94(\mathrm{~d}, J=8.5 \mathrm{~Hz}), 130.79$ (s), $133.91,154.27,158.90(\mathrm{~d}, J=248.2 \mathrm{~Hz}), 168.33(\mathrm{~d}, J=26.8$ $\mathrm{Hz}), 190.33$ (d, $J=25.7 \mathrm{~Hz})$; HPLC $(85: 15, n$-hexane $: i$ $\mathrm{PrOH}, 254 \mathrm{~nm}, 0.5 \mathrm{~mL} / \mathrm{min})$ Chiralpak IA column, $t_{\mathrm{R}}=24.0$ $\min$ (minor), $t_{\mathrm{R}}=38.0 \mathrm{~min}$ (major), $76 \%$ ee.

(2S, 3S)-Ethyl 2-Benzoyl-3-(tert-butoxycarbonylamino)2-fluoro-3-(3-nitrophenyl)propanoate (3g). Major diastereomer: $[\alpha]_{\mathrm{D}}^{31}=16.4\left(c=1.0, \mathrm{CHCl}_{3}\right) ;{ }^{1} \mathrm{H}$ NMR $(200$ $\left.\mathrm{MHz}, \mathrm{CDCl}_{3}\right) \delta 1.29(\mathrm{t}, J=6.9 \mathrm{~Hz}, 3 \mathrm{H}), 1.39(\mathrm{~s}, 9 \mathrm{H}), 4.17-$ $4.44(\mathrm{~m}, 2 \mathrm{H}), 5.62(\mathrm{~d}, J=10.3 \mathrm{~Hz}, 1 \mathrm{H}), 6.09\left(\mathrm{dd},{ }^{1} J=28.4\right.$, $\left.{ }^{2} J=10.3 \mathrm{~Hz}, 1 \mathrm{H}\right), 7.36-7.60(\mathrm{~m}, 4 \mathrm{H}), 7.79-7.90(\mathrm{~m}, 3 \mathrm{H})$, 8.10-8.14 (m, 1H), 8.30-8.31 (m, 1H); ${ }^{13} \mathrm{C} \mathrm{NMR} \mathrm{(50} \mathrm{MHz,}$ $\left.\mathrm{CDCl}_{3}\right) \delta 13.80,28.80,56.60(\mathrm{~d}, J=18.4 \mathrm{~Hz}), 63.51,80.72$, $101.63(\mathrm{~d}, J=204.5 \mathrm{~Hz}), 123.13,123.75,128.62,129.18$, $125.51,129.63,134.32,135.48,138.65,148.09,154.18$, $164.98(\mathrm{~d}, J=26.6 \mathrm{~Hz}), 189.88(\mathrm{~d}, J=25.4 \mathrm{~Hz})$; HPLC ( $85: 15, n$-hexane : $i$-PrOH, $254 \mathrm{~nm}, 0.5 \mathrm{~mL} / \mathrm{min}$ ) Chiralpak IA column, $t_{\mathrm{R}}=19.7 \mathrm{~min}$ (minor), $t_{\mathrm{R}}=29.4 \mathrm{~min}$ (major), $79 \%$ ee.

(2S, 3S)-Ethyl 2-Benzoyl-3-(tert-butoxycarbonylamino)2-fluoro-3-(1-naphthyl)propanoate (3h). Major diastereomer: $[\alpha]_{\mathrm{D}}^{30}=18.4\left(c=1.0, \mathrm{CHCl}_{3}\right) ;{ }^{1} \mathrm{H}$ NMR $(200 \mathrm{MHz}$, $\left.\mathrm{CDCl}_{3}\right) \delta 1.33(\mathrm{t}, J=7.1 \mathrm{~Hz}, 3 \mathrm{H}), 1.39(\mathrm{~s}, 9 \mathrm{H}), 4.29-4.40(\mathrm{~m}$, $2 \mathrm{H}), 5.29(\mathrm{~d}, J=10.2 \mathrm{~Hz}, 1 \mathrm{H}), 6.84\left(\mathrm{dd},{ }^{1} J=28.0 \mathrm{~Hz},{ }^{2} J=\right.$ $10.2 \mathrm{~Hz}, 1 \mathrm{H}), 7.23-7.39$ (m, 3H), 7.47-7.54 (m, 3H), 7.59$7.64(\mathrm{~m}, 1 \mathrm{H}), 7.67-7.83(\mathrm{~m}, 4 \mathrm{H}), 8.44-8.48(\mathrm{~m}, 1 \mathrm{H}) ;{ }^{13} \mathrm{C}$ NMR $\left(50 \mathrm{MHz}, \mathrm{CDCl}_{3}\right) \delta 13.84,28.12,52.43(\mathrm{~d}, J=$ 17.7Hz), 63.40, 80.10, 102.45 (d, $J=204.8 \mathrm{~Hz}), 124.13$, $124.92,125.90,126.77,128.42,128.88,129.32,129.43$, $131.60,133.69,154.32,165.59$ (d, $J=27.6 \mathrm{~Hz}), 190.91$ (d, $J$ $=24.8 \mathrm{~Hz})$; HPLC (80:20, $n$-hexane : $i$-PrOH, $254 \mathrm{~nm}, 0.5$ $\mathrm{mL} / \mathrm{min}$ ) Regis Whelk-O1 column, $t_{\mathrm{R}}=13.2 \mathrm{~min}$ (minor), $t_{\mathrm{R}}$ $=16.3 \mathrm{~min}$ (major), $81 \%$ ee.

(2S,3S)-Ethyl 2-Benzoyl-3-(tert-butoxycarbonylamino)2-fluoro-3-(2-furanyl)propanoate (3i). Major diastereomer: $[\alpha]_{\mathrm{D}}^{28}=35.3\left(c=1.0, \mathrm{CHCl}_{3}\right) ;{ }^{1} \mathrm{H}$ NMR $\left(200 \mathrm{MHz}, \mathrm{CDCl}_{3}\right)$ $\delta 1.28(\mathrm{t}, J=7.0 \mathrm{~Hz}, 3 \mathrm{H}), 1.43(\mathrm{~s}, 9 \mathrm{H}), 4.18-4.38(\mathrm{~m}, 2 \mathrm{H})$, $5.35(\mathrm{~d}, J=10.7 \mathrm{~Hz}), 6.17(\mathrm{dd}, J=28.2,10.7 \mathrm{~Hz}, 1 \mathrm{H}), 6.24-$ $6.26(\mathrm{~m}, 2 \mathrm{H}), 7.27-7.29(\mathrm{~m}, 1 \mathrm{H}), 7.38-7.46(\mathrm{~m}, 2 \mathrm{H}), 7.53-$ $7.60(\mathrm{~m}, 1 \mathrm{H}), 7.90-7.94(\mathrm{~m}, 2 \mathrm{H}) ;{ }^{13} \mathrm{C} \mathrm{NMR}(50 \mathrm{MHz}$, $\left.\mathrm{CDCl}_{3}\right) \delta 13.79,28.15,52.07(\mathrm{~d}, J=19.7 \mathrm{~Hz}), 63.24,80.33$, 101.45 (d, $J=204.5 \mathrm{~Hz}), 108.84,110.30,128.51,129.49$, $129.61,133.90,142.37,149.35,154.32,164.90(\mathrm{~d}, J=26.6$ Hz), 190.39 (d, $J=25.2 \mathrm{~Hz})$; HPLC (85 : 15, $n$-hexane : $i$ $\mathrm{PrOH}, 254 \mathrm{~nm}, 0.5 \mathrm{~mL} / \mathrm{min}$ ) Chiralpak IA column, $t_{\mathrm{R}}=20.9$ $\min$ (minor), $t_{\mathrm{R}}=34.5$ min (major), $98 \%$ ee.

(2S, 3S)-Ethyl 2-Benzoyl-3-(tert-butoxycarbonylamino)2-fluoro-3-(2-thienyl)propanoate (3j). Major diastereomer: 
$[\alpha]_{\mathrm{D}}^{26}=54.7\left(\mathrm{c}=1.0, \mathrm{CHCl}_{3}\right) ;{ }^{1} \mathrm{H} \mathrm{NMR}\left(200 \mathrm{MHz}, \mathrm{CDCl}_{3}\right)$ $\delta 1.27(\mathrm{t}, J=7.0 \mathrm{~Hz}, 3 \mathrm{H}), 1.41(\mathrm{~s}, 9 \mathrm{H}) 4.17-4.40(\mathrm{~m}, 2 \mathrm{H})$, $5.36(\mathrm{~d}, J=10.4 \mathrm{~Hz}, 1 \mathrm{H}), 6.31(\mathrm{dd}, J=28.3,10.4 \mathrm{~Hz}, 1 \mathrm{H})$, 6.86-6.90 (m, 1H), 7.06-7.08 (m, 1H), 7.16-7.18 (m, 1H), 7.36-7.44 (m, 2H), 7.51-7.59 (m, 1H), 7.89-7.93 (m, 2H); ${ }^{13} \mathrm{C}$ NMR $\left(50 \mathrm{MHz}, \mathrm{CDCl}_{3}\right) \delta 13.74,28.10,53.53(\mathrm{~d}, J=$ $19.35 \mathrm{~Hz}), 63.12,80.27,101.92(\mathrm{~d}, J=204.2 \mathrm{~Hz}), 125.53$, $126.56,127.18,128.46,129.45,129.56,133.91,138.75$, 154.11, 164.96 (d, $J=26.9 \mathrm{~Hz}), 190.34(\mathrm{~d}, J=25.3 \mathrm{~Hz})$; HPLC (90:10, $n$-hexane : $i-\mathrm{PrOH}, 254 \mathrm{~nm}, 0.5 \mathrm{~mL} / \mathrm{min})$ Chiralpak IA column, $t_{\mathrm{R}}=30.9 \mathrm{~min}\left(\right.$ minor), $t_{\mathrm{R}}=44.4 \mathrm{~min}$ (major), $95 \%$ ee.

(2S, 3S)-Ethyl 2-(4-nitrobenzoyl)-3-(tert-butoxycarbonylamino)-2-fluoro-3-(2-furanyl)propanoate (3k). Major diastereomer: $[\alpha]_{\mathrm{D}}^{31}=19.7\left(c=1.0, \mathrm{CHCl}_{3}\right) ;{ }^{1} \mathrm{H}$ NMR $(200$ $\left.\mathrm{MHz}, \mathrm{CDCl}_{3}\right) \delta 1.31(\mathrm{t}, J=7.2 \mathrm{~Hz}, 3 \mathrm{H}), 1.44(\mathrm{~s}, 9 \mathrm{H}), 4.20-$ $4.41(\mathrm{~m}, 2 \mathrm{H}), 5.36(\mathrm{~d}, J=10.2 \mathrm{~Hz}, 1 \mathrm{H}), 6.15(\mathrm{dd}, J=28.2$, $10.2 \mathrm{~Hz}, 1 \mathrm{H}), 6.27-6.31(\mathrm{~m}, 2 \mathrm{H}), 7.30(\mathrm{~m}, 1 \mathrm{H}), 8.01-8.06$ (m, 2H), 8.24-8.28 (m, 2H); ${ }^{13} \mathrm{C}$ NMR $\left(50 \mathrm{MHz}, \mathrm{CDCl}_{3}\right) \delta$ 13.83, 28.16, 52.21 (d, $J=19.3 \mathrm{~Hz}), 63.69,80.67,101.58$ (d, $J=204.4 \mathrm{~Hz}), 109.13,110.48,123.62,130.64,138.59$, $142.66,148.90,150.51,154.30,164.15(\mathrm{~d}, J=26.7 \mathrm{~Hz})$, $189.96(\mathrm{~d}, J=25.7 \mathrm{~Hz})$; HPLC ( $85: 15, n$-hexane $: i$-PrOH, $254 \mathrm{~nm}, 0.5 \mathrm{~mL} / \mathrm{min}$ ) Chiralpak IA column, $t_{\mathrm{R}}=26.8 \mathrm{~min}$ (minor), $t_{\mathrm{R}}=45.6 \mathrm{~min}$ (major), $98 \%$ ee.

$(2 S, 3 S)$-Ethyl 2-(4-(trifluoromethyl)phenyl)-3-(tertbutoxycarbonylamino)-2-fluoro-3-(2-furanyl)propanoate (31). Major diastereomer: $[\alpha]_{\mathrm{D}}^{30}=26.0\left(c=1.0, \mathrm{CHCl}_{3}\right) ;{ }^{1} \mathrm{H}$ NMR $\left(200 \mathrm{MHz}, \mathrm{CDCl}_{3}\right) \delta 1.30$ (t, $\left.J=7.3 \mathrm{~Hz}, 3 \mathrm{H}\right), 1.44$ (s, 9H), 4.20-4.40 (m, 2H), 5.35 (d, $J=10.5 \mathrm{~Hz}, 1 \mathrm{H}), 6.17(\mathrm{dd}$, $J=28.4,10.5 \mathrm{~Hz}, 1 \mathrm{H}), 6.25-6.27(\mathrm{~m}, 2 \mathrm{H}), 7.27-7.29(\mathrm{~m}$, $1 \mathrm{H}), 7.67-7.71(\mathrm{~m}, 2 \mathrm{H}), 7.99-8.03(\mathrm{~m}, 2 \mathrm{H}) ;{ }^{13} \mathrm{C}$ NMR $(50$ $\left.\mathrm{MHz}, \mathrm{CDCl}_{3}\right) \delta 13.79,28.14,52.14(\mathrm{~d}, J=19.2 \mathrm{~Hz}), 63.51$, $80.53,101.54$ (d, $J=204.5 \mathrm{~Hz}), 109.01,110.39,123.35$ (q, $J$ $=271.53 \mathrm{~Hz}), 125.55,129.80,134.94(\mathrm{q}, J=32.5 \mathrm{~Hz})$, 136.66, 142.55, 149.07, 154.31, $164.42(\mathrm{~d}, J=26.6 \mathrm{~Hz})$, $189.56(\mathrm{~d}, J=29.0 \mathrm{~Hz})$; HPLC ( $85: 15, n$-hexane $: i-\mathrm{PrOH}$, $254 \mathrm{~nm}, 0.5 \mathrm{~mL} / \mathrm{min}$ ) Chiralpak IA column, $t_{\mathrm{R}}=15.7 \mathrm{~min}$ (minor), $t_{\mathrm{R}}=28.5 \mathrm{~min}$ (major), $96 \%$ ee.

$(2 S, 3 S)$-Ethyl 2-(4-methoxybenzoyl)-3-(tert-butoxycarbonylamino)-2-fluoro-3-(2-furanyl)propanoate (3m). Major diastereomer: $[\alpha]_{\mathrm{D}}^{30}=19.1\left(c=1.0, \mathrm{CHCl}_{3}\right) ;{ }^{1} \mathrm{H}$ NMR (200 MHz, CDCl $) \delta 1.26$ (t, $J=6.9 \mathrm{~Hz}, 3 \mathrm{H}), 1.43$ (s, $9 \mathrm{H}), 3.84(\mathrm{~s}, 3 \mathrm{H}), 4.16-4.36(\mathrm{~m}, 2 \mathrm{H}), 5.36(\mathrm{~d}, J=10.5 \mathrm{~Hz}$, $1 \mathrm{H}), 6.16(\mathrm{dd}, J=28.3,10.5 \mathrm{~Hz}), 6.23-6.25(\mathrm{~m}, 2 \mathrm{H}), 6.86-$ $6.93(\mathrm{~m}, 2 \mathrm{H}), 7.79(\mathrm{~m}, 1 \mathrm{H}), 7.95-8.00(\mathrm{~m}, 2 \mathrm{H}) ;{ }^{13} \mathrm{C}$ NMR $\left(50 \mathrm{MHz}, \mathrm{CDCl}_{3}\right) \delta 13.74,28.10,51.96(\mathrm{~d}, J=19.5 \mathrm{~Hz})$, $55.42,63.06,80.19,101.50(\mathrm{~d}, J=204.4 \mathrm{~Hz}), 108.70$, $110.24,113.79,126.60,132.25,142.22,149.57,154.31$, $164.18,165.20(\mathrm{~d}, J=26.9 \mathrm{~Hz}), 188.24(\mathrm{~d}, J=24.3 \mathrm{~Hz})$; HPLC ( $85: 15, n$-hexane : $i$-PrOH, $254 \mathrm{~nm}, 0.5 \mathrm{~mL} / \mathrm{min}$ ) Chiralpak IA column, $t_{\mathrm{R}}=26.6 \mathrm{~min}\left(\right.$ minor), $t_{\mathrm{R}}=49.6 \mathrm{~min}$ (major), 96\% ee.

(2S, 3S)-Ethyl 2-(4-bromobenzoyl)-3-(tert-butoxycarbonylamino)-2-fluoro-3-(2-furanyl)propanoate (3n). Major diastereomer: $[\alpha]_{\mathrm{D}}^{31}=21.1\left(\mathrm{c}=1.0, \mathrm{CHCl}_{3}\right) ;{ }^{1} \mathrm{H}$ NMR $(200$ $\left.\mathrm{MHz}, \mathrm{CDCl}_{3}\right) \delta 1.28$ (t, $\left.J=7.0 \mathrm{~Hz}, 3 \mathrm{H}\right), 1.43(\mathrm{~s}, 9 \mathrm{H}), 4.19-$
4.37 (m, 2H), $5.32(\mathrm{~d}, J=10.1 \mathrm{~Hz}, 1 \mathrm{H}), 6.14(\mathrm{dd}, J=28.4$, $10.1 \mathrm{~Hz}, 1 \mathrm{H}), 6.23-6.31(\mathrm{~m}, 2 \mathrm{H}), 7.27-7.29(\mathrm{~m}, 1 \mathrm{H}), 7.54-$ $7.59(\mathrm{~m}, 2 \mathrm{H}), 7.78-7.81(\mathrm{~m}, 2 \mathrm{H}) ;{ }^{13} \mathrm{C}$ NMR $(50 \mathrm{MHz}$, $\left.\mathrm{CDCl}_{3}\right) \delta 13.81,28.16,52.08$ (d, $\left.J=19.3 \mathrm{~Hz}\right), 63.40,80.45$, 101.51 (d, $J=204.1 \mathrm{~Hz}), 108.93,110.36,129.47,131.00$, 131.92, 132.48, 142.46, 149.22, 154.31, 164.68 (d, $J=27.9$ $\mathrm{Hz}), 189.64(\mathrm{~d}, J=25.5 \mathrm{~Hz})$; HPLC $(90: 10, n$-hexane : $i$ PrOH, $254 \mathrm{~nm}, 0.5 \mathrm{~mL} / \mathrm{min}$ ) Chiralpak IA column, $t_{\mathrm{R}}=24.7$ $\min$ (minor), $t_{\mathrm{R}}=55.2 \mathrm{~min}$ (major), $98 \%$ ee.

$(S)$-Ethyl 2-((S)-(tert-butoxycarbonylamino)(p-tolyl)methyl)-2-fluoro-3-oxobutanoate (30). Mixture of diastereomers: $[\alpha]_{\mathrm{D}}^{27}=9.5\left(c=1.0, \mathrm{CHCl}_{3}\right) ;{ }^{1} \mathrm{H}$ NMR $(200$ $\left.\mathrm{MHz}, \mathrm{CDCl}_{3}\right) \delta 1.35-1.26(\mathrm{~m}, 4.15 \mathrm{H}), 1.38(\mathrm{~m}, 12.46 \mathrm{H})$, $2.00(\mathrm{~d}, J=5.1 \mathrm{~Hz}, 3 \mathrm{H}), 2.23(\mathrm{~m}, 4.15 \mathrm{H}), 2.34(\mathrm{~d}, J=5.1$ $\mathrm{Hz}, 1.15 \mathrm{H}), 4.02-4.13(\mathrm{~m}, 0.77 \mathrm{H}) 4.18-4.35(\mathrm{~m}, 2.0 \mathrm{H}), 5.50$ $(\mathrm{m}, 1.38 \mathrm{H}), 5.70(\mathrm{~m}, 1.38 \mathrm{H}), 7.26-7.10(\mathrm{~m}, 5.54 \mathrm{H}) ;{ }^{13} \mathrm{C}$ NMR $\left(50 \mathrm{MHz}, \mathrm{CDCl}_{3}\right) \delta 13.71,13.91,26.43 \times 2,28.16 \times 2$, $28.16 \times 2,55.97(\mathrm{~d}, J=17.9 \mathrm{~Hz}), 56.73(\mathrm{~d}, J=17.9 \mathrm{~Hz})$, 62.76, 63.03, 79.22×2, 88.93 (d, $J=201.33 \mathrm{~Hz}), 101.98$ (d, $J$ $=201.33 \mathrm{~Hz}), 127.94 \times 2,128.16,128.20,129.25 \times 2,138.25 \times 2$, 154.28x2, $165.48 \times 2$ (d, $J=26.9 \mathrm{~Hz}), 200.09 \times 2$ (d, $J=25.6$ $\mathrm{Hz})$; HPLC (90:10, $n$-hexane : $i$-PrOH, $220 \mathrm{~nm}, 1.0 \mathrm{~mL} /$ min) Chiralpak IC column, $t_{\mathrm{R}}=6.7 \mathrm{~min}\left(\right.$ minor), $t_{\mathrm{R}}=9.4$ $\min$ (major), $80 \%$ ee.

(S)-Benzyl 2-((S)-(tert-butoxycarbonylamino)(p-tolyl)methyl)-2-fluoro-3-oxobutanoate (3p). Major diastereomer: $[\alpha]_{\mathrm{D}}^{27}=-2.4\left(c=1.0, \mathrm{CHCl}_{3}\right) ;{ }^{1} \mathrm{H}$ NMR $(200 \mathrm{MHz}$, $\left.\mathrm{CDCl}_{3}\right) \delta 1.36(\mathrm{~s}, 9 \mathrm{H}), 1.99(\mathrm{~d}, J=4.41 \mathrm{~Hz}, 3 \mathrm{H}), 2.31(\mathrm{~s}$, $3 \mathrm{H}$ ), 5.31-5.14 (m, 2H), 5.51 (dd. ${ }^{1} J=20.85,{ }^{2} J=10.14$, 2H), 7.26-7.04 (m, 4H), 7.34-7.30 (m. 5H); ${ }^{13} \mathrm{C}$ NMR (50 $\left.\mathrm{MHz}, \mathrm{CDCl}_{3}\right) \delta 21.09,26.35,28.15,56.79(\mathrm{~d}, J=18.05 \mathrm{~Hz})$, $68.35,80.29,103.00(\mathrm{~d}, J=203.75 \mathrm{~Hz}), 128.15,128.52$, $128.61,129.26,132.58,132.84,134.57,138.27,154.35$, 164.21 (d, $J=26.9 \mathrm{~Hz}), 199.60$ (d, $J=25.6 \mathrm{~Hz}) ;$ HPLC $(90$ : 10, $n$-hexane : $i$-PrOH, $220 \mathrm{~nm}, 1.0 \mathrm{~mL} / \mathrm{min})$ Chiralpak IC column, $t_{\mathrm{R}}=7.4 \mathrm{~min}$ (minor), $t_{\mathrm{R}}=10.0 \mathrm{~min}$ (major), $72 \%$ ee.

\section{References}

1. (a) Hudlicky, M.; Pavlath, A. E. Chemistry of Organic Fluorine Compounds II; American Chemical Society: Washington, DC, 1995. (b) Kirk, K. L. J. J. Fluorine Chem. 2006, 127, 1013. (c) Isanobor, C.; O'Hagan, D. J. Fluorine Chem. 2006, 127, 303. (d) Muller, K.; Faeh, C.; Diederich, F. Science 2007, 317, 1881. (e) Kirk, K. L. Org. Process Res. Dev. 2008, 12, 305.

2. (a) Hiyama, T.; Kanie, K.; Kusumoto, T.; Morizawa, Y.; Shimizu, M. Organofluorine Compounds: Chemistry and Applications; Springer-Verlag: Berlin, 2000. (b) Purser, S.; Moore, P. R.; Swallow, S.; Gouverneur, V. Chem. Soc. Rev. 2008, 37, 320.

3. (a) Enantiocontrolled Synthesis of Fluoro-organic Compounds; Soloshonok, V. A., Ed.; John Wiley \& Sons: Chichester, 1999. (b) Ramachandran, P. V., Ed.; Asymmetric Fluoroorganic Chemistry: Synthesis, Application, and Future Directions; ACS Symposium Series 746; American Chemical Society: Washington, DC, 2000.

4. For reviews, see: (a) Mikami, K.; Itoh, Y.; Yamanaka, M. Chem. Rev. 2004, 104, 1. (b) Ibrahim, H.; Togni, A. Chem. Commun. 2004, 1147. (c) Ma, J.-A.; Cahard, D. Chem. Rev. 2008, 108, PR1. (d) France, S.; Weatherwax, A.; Lectka, T. Eur. J. Org. Chem. 2005, 475. (e) Oestreich, M. Angew. Chem. Int. Ed. 2005, 44, 2324. (f) Pihko, P. M. Angew. Chem. Int. Ed. 2006, 45, 544. (g) 
Prakash, G. K. S.; Beier, P. Angew. Chem. Int. Ed. 2006, 45, 2172. (h) Bobbio, C.; Gouverneur, V. Org. Biomol. Chem. 2006, 4, 2065. (i) Shibata, N.; Ishimaru, T.; Nakamura, S.; Toru, T. J. Fluorine Chem. 2007, 128, 469. (j) Brunet, V. A.; O'Hagan, D. Angew. Chem. Int. Ed. 2008, 47, 1179. (k) Smits, R.; Cadicamo, C. D.; Burger, K.; Koksch, B. Chem. Soc. Rev. 2008, 37, 1727. (1) Kang, Y. K.; Kim, D. Y. Curr. Org. Chem. 2010, 14, 917.

5. For recent selected examples of catalytic asymmetric fluorinations of active methines, see: (a) Hintermann, L.; Togni, A. Angew. Chem. Int. Ed. 2000, 39, 4359. (b) Kim, D. Y.; Park, E. J. Org. Lett. 2002, 4, 545. (c) Hamashima, Y.; Yagi, K.; Takano, H.; Tamás, L.; Sodeoka, M. J. Am. Chem. Soc. 2002, 124, 14530. (d) Ma, J.-A.; Cahard, D. Tetrahedron: Asymmetry 2004, 15, 1007. (e) Shibata, N.; Ishimaru, T.; Nagai, T.; Kohno. J.; Toru, T. Synlett 2004, 1703. (f) Bernardi, L.; Jørgensen, K. A. Chem. Commun. 2005, 1324. (g) Kim, S. M.; Kim, H. R.; Kim, D. Y. Org. Lett. 2005, 7, 2309. (h) Kim, H. R.; Kim, D. Y. Tetrahedron Lett. 2005, 46, 3115. (i) Ishimaru, T.; Shibata, N.; Horikawa, T.; Yasuda, N.; Nakamura, S.; Toru, T.; Shiro, M. Angew. Chem. Int. Ed. 2008, 47, 4157. (j) Lee, N. R.; Kim, S. M.; Kim, D. Y. Bull. Korean Chem. Soc. 2009, 30, 829. (k) Kang, S. H.; Kim, D. Y. Adv. Synth. Catal. 2010, 352, 2783 .

6. For asymmetric Michael-type reactions of $\alpha$-fluoromalonates, see: (a) Kim, D. Y.; Kim, S. M.; Koh, K. O.; Mang, J. Y. Bull. Korean Chem. Soc. 2003, 24, 1425. (b) Nichols, P. J.; DeMattei, J. A.; Barnett, B. R.; LeFur, N. A.; Chuang, T.-H.; Piscopio, A. D.; Koch, K. Org. Lett. 2006, 8, 1495. (c) Kwon, B. K.; Kim, S. M.; Kim, D. Y. J. Fluorine Chem. 2009, 130, 759. (d) Companyo, X.; Hejnova, M.; Kamlar, M.; Vesely, J.; Moyano, A.; Rios, R. Tetrahedron Lett. 2009, 50, 5051.

7. For asymmetric Michael-type reactions of $\alpha$-fluoro- $\beta$-ketoesters, see: (a) Nakamura, M.; Hajra, A.; Endo, K.; Nakamura, E. Angew. Chem. Int. Ed. 2005, 44, 7248. (b) He, R.; Wang, X.; Hashimoto, T.; Maruoka, K. Angew. Chem. Int. Ed. 2008, 47, 9466. (c) Mang, J. Y.; Kwon, D. G.; Kim, D. Y. J. Fluorine Chem. 2009, 130, 259. (d) Han, X.; Luo, J.; Liu, C.; Lu, Y. Chem. Commun. 2009, 2044. (e) Li, H.; Zhang, S.; Yu, C.; Song, X.; Wang, W. Chem. Commun. 2009, 2136. (f) Oh, Y.; Kim, S. M.; Kim, D. Y. Tetrahedron Lett. 2009, 50, 4674. (g) Ishimaru, T.; Ogawa, S.; Tokunaga, E.; Nakamura, S.; Shibata, N. J. Fluorine Chem. 2009, 130, 1049. (h) Cui, H.-F.; Yang, Y.-Q.; Chai, Z.; Li, P.; Zheng, C.-W.; Zhu, S.-Z. J. Org. Chem. 2010, 75, 117.

8. For asymmetric reactions using fluorobis(phenysulfonyl)methane derivatives, see: (a) Fukuzumi, T.; Shibata, N.; Sugiura, M.; Yasui, H.; Nakamura, S.; Toru, T. Angew. Chem. Int. Ed. 2006, 45, 4973. (b) Mizuta, S.; Shibata, N.; Goto, Y.; Furukawa, T.; Nakamura, S.; Toru, T. J. Am. Chem. Soc. 2007, 129, 6394. (c) Furukawa, T.; Shibata, N.; Mizuta, S.; Nakamura, S.; Toru, T.; Shiro, M. Angew. Chem. Int. Ed. 2008, 47, 8051. (d) Moon, H. W.; Cho, M. J.; Kim, D. Y. Tetrahedron Lett. 2009, 50, 4896. (e) Furukawa, T.; Goto, Y.; Kawazoe, J.; Tokunaga, E.; Nakamura, S.; Yang, Y.; Du, H.; Kakehi, A.; Shiro, M.; Shibata, N. Angew. Chem. Int. Ed. 2010, 49,1642

9. For selected recent reviews, see: (a) Verkade, J. M. M.; van Hemert, L. J. C.; Quaedflieg, P. J. L. M.; Rutjes, F. P. J. T. Chem. Soc. Rev. 2008, 37, 29. (b) Ting, A.; Schaus, S. E. Eur. J. Org. Chem. 2007, 5797. (c) Marques, M. M. B. Angew. Chem. Int. Ed. 2006, 45, 348. (d) Cordova, A. Acc. Chem. Res. 2004, 37, 102.
10. Selected examples of Mannich-type reactions of enolates, see: (a) Sikert, M.; Schneider, C. Angew. Chem., Int. Ed. 2008, 47, 3631. (b) Itoh, J.; Fuchibe, K.; Akiyama, T. Synthesis 2008, 1319. (c) Kobayashi, S.; Yazaki, R.; Seki, K.; Ueno, M. Tetrahedron 2007, 63, 8425. (d) Saruhashi, K.; Kobayashi, S. J. Am. Chem. Soc. 2006, 128, 11232. (e) Kobayashi, S.; Ueno, M.; Saito, S.; Mizuki, Y.; Ishitani, H.; Yamashita, Y. Proc. Natl. Acad. Sci. U.S.A. 2004, 101, 5476. (f) Akiyama, T.; Itoh, J.; Yokota, K.; Fuchibe, K. Angew. Chem., Int. Ed. 2004, 43, 1566. (g) Wenzel, A. G.; Jacobsen, E. N. J. Am. Chem. Soc. 2002, 124, 12964.

11. (a) Hamashima, Y.; Sasamoto, N.; Umebayashi, N.; Sodeoka, M. Chem. Asian J. 2008, 3, 1443. (b) Chen, Z.; Morimoto, H.; Matsunaga, S.; Shibasaki, M. J. Am. Chem. Soc. 2008, 130, 2170. (c) Kobayashi, S.; Gustafsson, T.; Shimizu, Y.; Kiyohara, H.; Matsubara, R. Org. Lett. 2006, 8, 4923. (d) Hamashima, Y.; Sasamoto, N.; Hotta, D.; Somei, H.; Umebayashi, N.; Sodeoka, M. Angew. Chem., Int. Ed. 2005, 44, 1525. (e) Kang, Y. K.; Kim, D. Y. J. Org. Chem. 2009, 74, 5734. (f) Lee, J. H.; Kim, D. Y. $A d v$. Synth. Catal. 2009, 351, 1779. (g) Kim, E. J.; Kang, Y. K.; Kim, D. Y. Bull. Korean Chem. Soc. 2009, 30, 1437. (h) Lee, J. H.; Kim, D. Y. Synthesis 2010, 1860.

12. (a) Han, X.; Kwiatkowski, J.; Xue, F.; Huang, K.-W.; Lu, Y. Angew. Chem., Int. Ed. 2009, 48, 7604. (b) Jiang, Z.; Pan, Y.; Zhao, Y.; Ma, T.; Lee, R.; Yang, Y.; Huang, K.-W.; Wong, M. W.; Tan, C.-H. Angew. Chem., Int. Ed. 2009, 48, 3627. (c) Pan, Y; Zhao, Y.; Ma, T.; Yang, Y.; Liu, H.; Jiang, Z.; Tan, C.-H. Chem. Eur. J. 2010, 16, 779.

13. (a) Kim, D. Y.; Huh, S. C.; Kim, S. M. Tetrahedron Lett. 2001, 42 6299. (b) Kim, D. Y.; Huh, S. C. Tetrahedron 2001, 57, 8933. (c) Park, E. J.; Kim, M. H.; Kim, D. Y. J. Org. Chem. 2004, 69, 6897. (d) Kang, Y. K.; Kim, D. Y. Tetrahedron Lett. 2006, 47, 4565. (e) Kang, Y. K.; Cho, M. J.; Kim, S. M.; Kim, D. Y. Synlett 2007, 1135. (f) Cho, M. J.; Kang, Y. K.; Lee, N. R.; Kim, D. Y. Bull. Korean Chem. Soc. 2007, 28, 2191. (g) Kim, S. M.; Kang, Y. K.; Cho, M. J.; Mang, J. Y.; Kim, D. Y. Bull. Korean Chem. Soc. 2007, 28, 2435. (h) Lee, J. H.; Bang, H. T.; Kim, D. Y. Synlett 2008, 1821. (i) Kang, Y. K.; Kim, D. Y. Bull. Korean Chem. Soc. 2008, 29, 2093 (j) Kim, D. Y. Bull. Korean Chem. Soc. 2008, 29 , 2036. (k) Mang, J. Y.; Kwon, D. G.; Kim, D. Y. Bull. Korean Chem. Soc. 2009, 30, 249. (1) Kang, S. H.; Kang, Y. K.; Kim, D. Y. Tetrahedron 2009, 65, 5676. (m) Kang, Y. K.; Kim, S. M.; Kim, D. Y. J. Am. Chem. Soc. 2010, 132, 11847.

14. (a) Kim, S. M.; Lee, J. H.; Kim, D. Y. Synlett 2008, 2659. (b) Jung, S. H.; Kim, D. Y. Tetrahedron Lett. 2008, 49, 5527. (c) Mang, J. Y.; Kim, D. Y. Bull. Korean Chem. Soc. 2008, 29, 2091. (d) Kwon, B. K.; Kim, D. Y. Bull. Korean Chem. Soc. 2009, 30, 1441. For preliminary communication of our studies of Mannichtype reaction of $\alpha$-fluoro- $\beta$-ketoesters, see: (e) Yoon, S. J.; Kang, Y. K.; Kim, D. Y. Synlett 2011, 420. (f) Kang, Y. K.; Kim, D. Y. Tetrahedron Lett. 2011, 52, 2356.

15. (a) Brunner, H.; Buegler, J.; Nuber, B. Tetrahedron: Asymmetry 1995, 6, 1699. (b) Oliva, C. G.; Silva, A. M. S.; Resende, D. I. S. P.; Paz, F. A. A.; Cavaleiro, J. A. S. Eur. J. Org. Chem. 2010, 3449. (c) Arai, T.; Watanabe, M.; Fujiwara, A.; Yokoyama, N.; Yanagisawa, A. Angew. Chem. Int. Ed. 2006, 45, 6978. (d) Arai, T.; Watanabe, M.; Yanagisawa, Org. Lett. 2007, 9, 3595. (e) Liu, Q.-Z.; Wang, X.-L.; Luo, S.-W.; Zheng, B. L.; Qin, D.-B.; Tetrahedron Lett. 2008, 49, 7434. 